\title{
THE TREATMENT OF URETHRAL STRICTURE BY EXCISION.
}

\section{Bx R. hamilton RUSSell, Melbourne.}

Foreword.-The method of operation described in the following paper is based on a fact which up to three or. four years ago was quite unknown to me, and is to the best of my belief not generally recognized. The fact is that the male urethra may be slit up from the membranous portion to the meatus (with, of course, division of the scrotum in such a case), or to a less extent, with the assurance that no harm will result, and that no difficulty will be experienced in restoring its integrity, provided efficient perineal drainage of the bladder is secured during healing. In ordinary single stricture of the bulbous urethra it is not necessary to invade the scrotum; an exposure of three or four inches of the channel, from the membranous urethra forwards, can be readily secured, and this is usually sufficient.

The urethra when slit up is converted from a tube lined with mucous membrane into a 'riband' covered with mucous membrane; its restoration is to be left entirely to natural processes, and will depend upon the obvious fact that a strip of mucous membrane flanked on either side by raw tissues which tend naturally to fall together and cohere, will, when this has taken place, be of necessity re-converted into a tube.

Should the scrotum have to be divided and the penile urethra slit up, in a case of multiple stricture, the skin of the scrotum and penis must be sutured, but the urethra is left to take care of itself.

Finally, given a stricture at any point of the slit-up urethra, the damaged portion is exposed to plain view and can be readily excised, the two ends of the urcthral 'riband' being then brought together by five catgut sutures, after which it is left to convert itself into a tube in the way $I$ have described.

Wherever in the following paper, in reporting a case, it is stated that what I call the 'hypospadias operation' was performed, I wish it to be understood that I should not employ it now. It is an operation which has proved of incalculable value to me in suggesting the far better method which is here brought forward; but I see no reason to suppose that I shall ever perform it again.

In the following paper I shall endeavour to elaborate the foregoing general statement, show how the principles indicated above work out in actual practice, and report six of my own cases, together with two additional ones the notes of which have been supplied by the kindness of my colleague, Mr. Fay Maclure.

In the march of surgical progress the treatment of urethral stricture has always seemed to lag somewhat behind. The methods at present in use, 


\section{THE BRITISH JOURNAL OF SURGERY}

other than preventive methods, may be ranged into two classes: (1) Those that do not involve a perineal operation; and (2) Those that do.

The first group comprises : (a) Simple dilatation; (b) Internal urethrotomy. The second comprises : (c) External urethrotomy; (d) Excision of the stricture.

Between these two classes there is a great gulf fixed, for all surgeons of cxperience are agreed as to the immense advantage of avoiding an operation on the perineum; and the question whether there shall or shall not be a perineal operation is decided upon the issue as to whether or not it is possible to introduce an instrument through the stricture. If an instrument of any kind can be coaxed through, simple dilatation can be carried out, aided, if the surgeon chooses, by internal urethrotomy. If the stricture proves impassable, then a perineal operation has to be performed, which commonly takes the form of external urethrotomy after the method of Wheelhouse, or some similar procedure. Excision of the stricture has never been very generally practised, and is attempted in only a small minority of cases; the reason is that the operation, as usually performed, is difficult and uncertain in its results, and surgeons are a little shy of it, in the natural fear lest things should be made worse instead of better. External urethrotomy, which gives immediate relief in conditions of great urgency, has proved in a large number of cases to be elusive and disastrous in the long run, and to eventuate in the most intractable kind of cicatricial stricture. This is so well recognized by surgeons of experience in this class of work, that they urge that great pains should be taken to obviate the perineal open operation. Moreover, they are rather inclined to pride themselves on seldom or never being defeated by the difficulties offered by the most formidable stricture, so that they will expend long periods of time and much patience upon prolonged sittings under anæsthesia, in the endcavour to find a way past the stricture for a fine bougie. For many years $I$ have held and acted upon such views, although I must confess that I have occasionally been beaten by a tight stricture, despite prolonged attempts under anæsthesia conducted with what seemed to me exemplary patience, and at last have been driven to external urethrotomy, which in such a case means evading a present difficulty at the price of aggravating it in the future-a very unsatisfactory piece of surgery indeed.

I desire to bring before the profession a simple and safe method of performing the radical operation of excision of urethral stricture which $I$ have practised for the last three or four years with great satisfaction. It has not only entirely superseded the operation of external urethrotomy in my practice, but the results have been such as to even quench my enthusiasm for prolonged and patient attempts at getting an instrument through a tight stricture; so that I am now distinctly of opinion that to find a way through a stricture of any severity is of very questionable benefit to the patient; rather will he be more fortunate in having it excised than in having it dilated. Let me explain that this method does not entirely do away with the use of dilating instruments after the operation; but their use is reduced so much, and the part they are required to play, which is in the main precautionary, is so easy of accomplishment and so light, that it really bears no resemblance to their employment in cases where dilatation of a stricture of even moderate severity is being undertaken. 


\section{TREATMENT OF URETHRAL STRICTURE}

I shall probably succeed best in.making the whole matter clear by briefly stating the history of the operation, and the manner in which I was led to its performance in the first instance. In the Australian Medical Journal of December 23, 1911, I published a suggestion for mitigating the sufferings of patients with very intractable stricture by an operation which laid open the perineal and scrotal portions of the urethra, thus simulating artificially the main features of perineoscrotal hypospadias. The urethra was opened in the perineum, and slit up from the bulb forwards as far as the root of the scrotum; the scrotum was also divided, and the testes were each sewn up in a separate scrotal bag; the scrotal portion of the urethra was slit up as far as the root of the penis. The man had in future to pass water in the feminine manner.

I instanced two examples in which this plan had been carried out with very great benefit. One obvious shortcoming attaching to this operation is that it may be impossible to get behind the stricture should it be very far back in the bulb. Even in such a case, however, I was hopeful that cxperience might prove the operation to be worth doing, as it would enable the surgeon to deal directly with the stricture, with the advantage of direct vision, which is a very different matter from dealing with it per urethram.

There is yet one more notable feature of the operation, which is this: if at any time it is desired to close the urethra and restore the channel to its normal state, to do this will be a very easy matter. Indeed the operation arose, in the first instance, at a time when my thoughts were much directed to the subject of hypospadias, and I had conceived the project of first converting the subject of a bad stricture into a case of hypospadias, and then operating on him for that condition, making a new urethra independent of the stricture. My first patient, however, found such complete relief from the initial operation that he declined any further procedure for the repair of his urethra.

The following case is instructive, though it is not a case of stricture, but of complete severance of the urethra.

R. L. B., age 26, was admitted to the Alfred Hospital, November, 1910. Four weeks prior to admission he sustained a severe injury to the perineum by falling across an iron bar. Ever since the accident he had been passing water with great pain, difficulty, and bleeding; there was, however, no extravasation. There was a hard defined swelling about an inch in length in the course of the urethra in the perineum.

At the operation it was found that the urethra had been completely-divided, and the distal severed end doubled back upon itself into the urethral channel, causing the small but defined swelling that was felt externally, and leaving an inch or more of the urinary channel formed only by the external fibrous sheath of the urethra, which had evidently escaped laceration. In order to restore the urethra, the doubledback portion had to be fully slit up longitudinally, and also the end of the proximal torn portion; the ends were then adjusted, and sutured together with catgut. As I expected future trouble, and thought this was eminently a case in which great advantage might accrue from having immediate access to the anticipated stricture, I performed the hypospadias operation, and drained the bladder with a rubber catheter introduced through the perineum. To my great surprise, however, no stricture formed, and after waiting twelve months I repaired the scrotum and urethra with perfect ease, thus restoring the patient to his normal condition. In July, 1913, no instrument having been passed for two yeárs, a full-sized bougie passed without any hitch whatever. 


\section{THE BRITISH JOURNAL OF SURGERY}

The outstanding facts proclaimed by this case are: (1) The urethra may be slit up to any extent longitudinally, and repaired easily without damage ; (2) A simple annular cicatrix in the urethra has little or no tendency to produce troublesome stricture, provided the ends are neatly adjusted and sutured, and that the healing process is protected from disturbance by urine; (3) As a

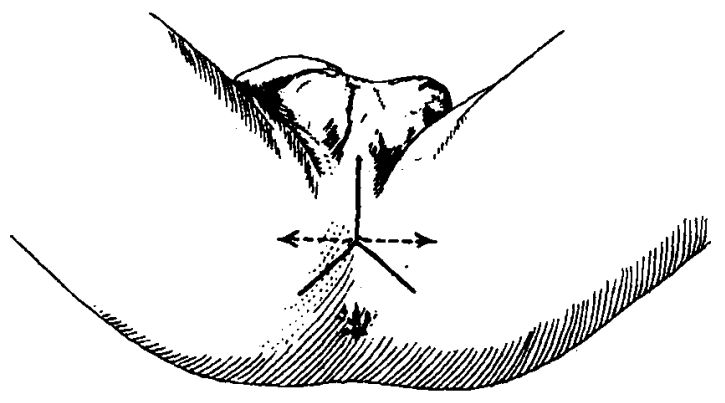

FIG. 204.-The perineal incisions. corollary of the two previous statements, the urethra may be freely slit up and converted into - a 'riband' instead of a tube, and can then be neatly and accurately sutured; and (4) This plan presents great advantages over the ordinary method of suturing the urethral tube over a catheter.

The formal operation is carried out in the extreme

lithotomy position, with the pelvis well raised, and is done in the following three stages :-

Stage 1.--Exposure and opening of the membranous urethra and slitting it up forwards towards the stricture.

Incision as for perineal prostatectomy-an inverted $\dot{\Lambda}$ having the apex at the central point of the perineum (Fig. 204, behind dotted line). The ischiorectal fossa is opened up on either side; and a bifid retractor used to draw the

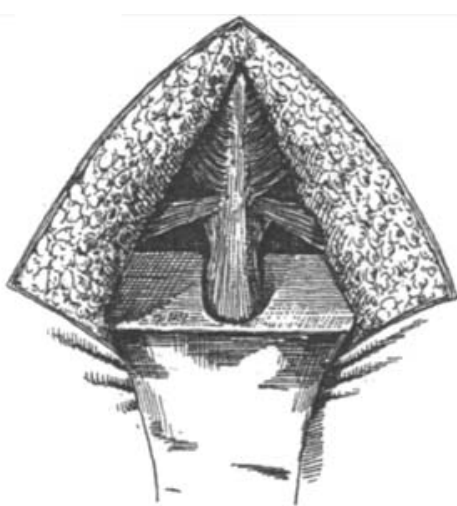

Fic. 205.-The central tendon and its attached muscles exposed: a bifid retractor draws back the external sphincter.

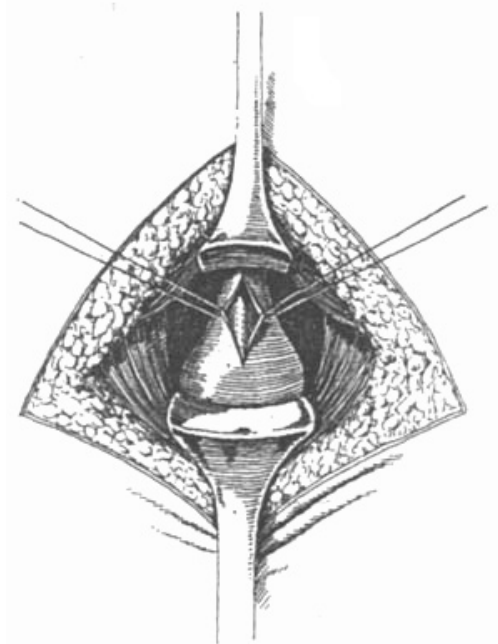

Fig. 206.-The membranous urethra slit up longitudinslly; silk thread retrac. tors have been introduced into the mar gins of the opening.

external sphincter backwards, while that muscle is detached at the central tendon connecting it with the bulbocavernosus muscle (Fig. 205); the bulb and the transverse perinei muscles are drawn forward, and the membranous 


\section{TREATMENT OF URETHRAL STRICTURE 379}

urethra and apex of the prostate exposed exactly as in the operation of perineal prostatectomy (Fig. 206). The membranous urethra is next opened longitudinally, a silk thread retractor introduced into either side of the opening, and the urethra then slit up forwards until the back of the stricture is encountered.

Stage 2.-Exposure and opening of the urethra in front of the stricture and slitting it up backwards towards the stricture.

Median incision, meeting the apex of the former incision (Fig. 204, in front of the dotted lines). A director or Wheelhouse staff is passed, and the urethra opened upon it in front of the stricture. Silk thread retractors are introduced' into the margins of this opening also, and the urethra is slit up backwards to the stricture, so that the length of the urethra, including the strictured portion and an inch or two behind and in front of it, will be plainly exposed (Fig. 207).

Stage 3.-Excision of the stricture and suturing of the urethra.

The strictured portion of the urethra, together with the fibrous extra-urethral masses, is now excised completely; the cut ends are then loosened and freed by undercutting (Fig. 208), and brought together accurately by five interrupted sutures of catgut (Fig. 209).

Leaving the urethra without any further sutures, a rubber catheter is fastened in the bladder, and the two lateral incisions in the perineum are sutured with deep silkworm-gut sutures, two on either side (Fig. 210).

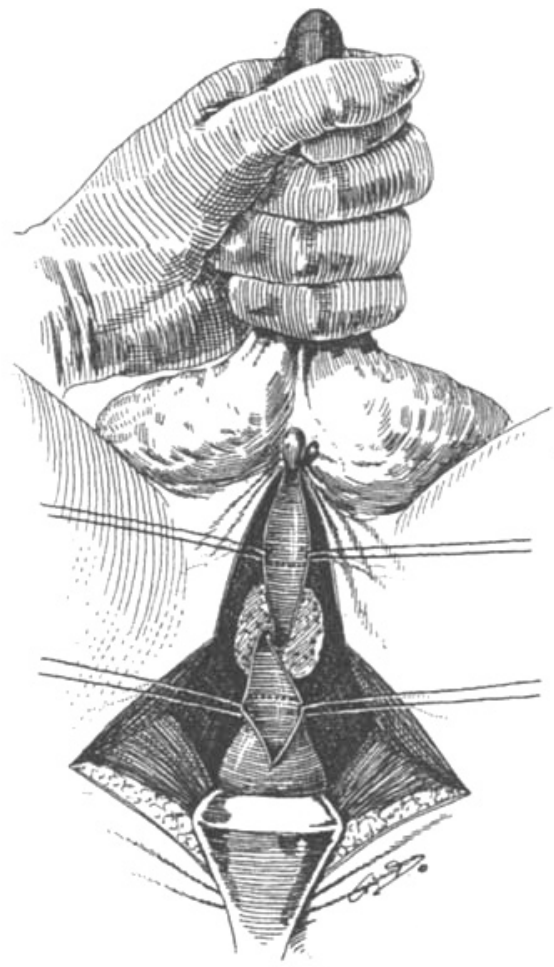

F1a. 207. - The urethra in front of the stricture opened or a director, and the stricture and its peri-urethral Gbrous masses exposed. No sutures are placed in the perineal wound in front of the catheter. The catheter must be left for at least a week; it may then be removed, and the perineal wound allowed to heal.

The foregoing sets forth briefly the steps of the operation; there are, however, one or two matters which should be alluded to in greater detail.

When the stricture is fully exposed in the operation, the following points must be specially noticed: (1) The peri-urethral masses of fibrous tissue which caused the obstruction; (2) The dilatation of the urethra behind the obstruction; (3) Owing to the pressure of the urine forward against the stricture, it will frequently be observed that the urethra, in addition to being dilated behind the stricture, will have become somewhat pushed to one side, so that a condition approaching to an S-curve appears in the channel at the point 


\section{THE BRITISH JOURNAL OF SURGERY}

of obstruction, and the directions of the urethral channels behind and in front of the stricture no longer correspond, but are out of alignment; in fact the posterior urethra tends to be pushed forward and to one side of the stricture, greatly aggravating the difficulty of micturition, and rendering the introduction

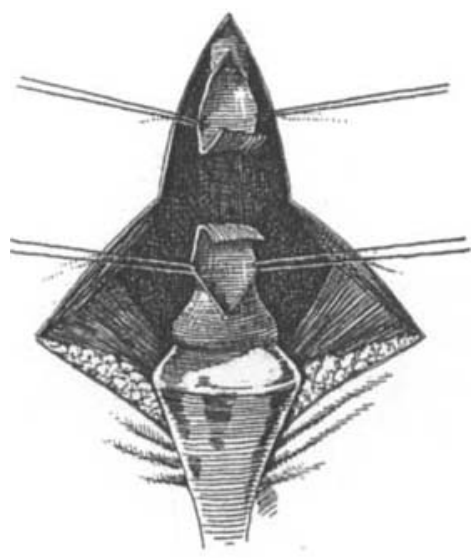

Fic. 208.-The stricture and the surrounding fibrous tissue have been ex. cised. and the cut ends of the urethra are loosened and fręed by undercutting.

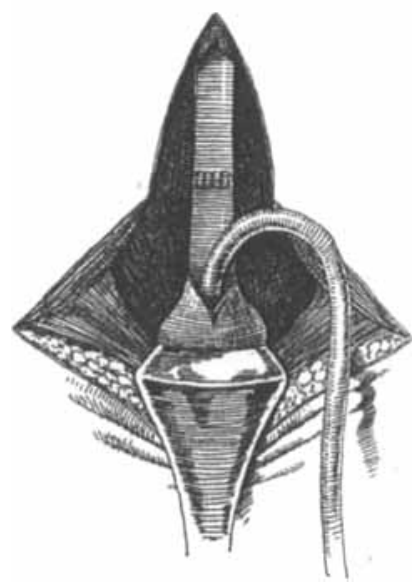

Fro. 209.-The cut ends united by five interrupted sutures.

of an instrument almost impossible. This is shown in a somewhat exaggerated way in Fig. 20\%.

Again, the surgeon must decide as to his exact procedure after the stricture is exposed to view; the urethra will appear as a 'strip,' interrupted and damaged at the seat of stricture, and the exact amount it will be necessary to

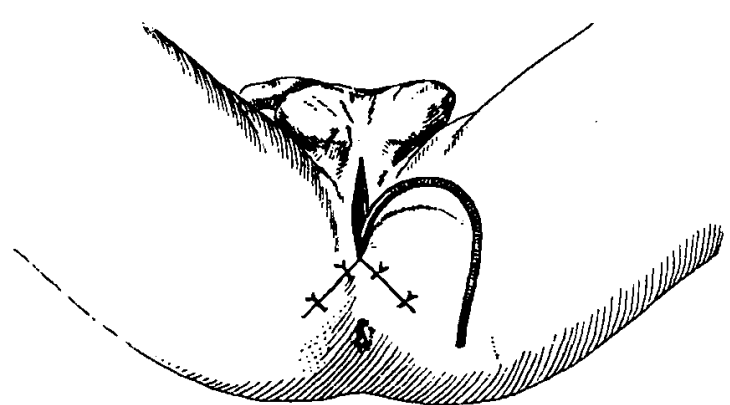

Fio. 210.-Suture of the lateral perineal incisions, the median wound being left open; a catheter is fastenod in the bladder.

removed upwards of an inch of the urethra.

The position of the catheter insures that the urethral wound which has been sutured shall be protected from contact with urine during healing; when the catheter is taken out it will be found that the wound will close very rapidly, and healing will be complete in a few days. In brief, the restoration of the membrane seems but little damaged, only the periurethral masses need be cut away. As a rule, however, it is necessary to excise a portion of ragged and injured mucous membrane. It will be found that the completeness of the exposure renders it easy to conserve the mucous membrane to the utmost; nevertheless I have on more than one occasion remove must be determined 


\section{TREATMENT OF URETHRAL STRICTURE 381}

urethral tube is left entirely to natural processes, as stated at the outset. When healing has taken place it is always found that the patient passes water naturally in a full stream.

Several weeks are allowed to elapse after the operation before an instrument is passed to ascertain the exact condition of the urethra at the point of suture. As a rule this spot can be felt, and but little more, with a good-sized bougie, and in any case it is at once dilated gently up to the full size. Lister's graduated instruments are always used. The further management of the case is very easy, and resolves itself into the occasional passage of a full-sized instrument as a precautionary measure, the intervals being quite long ; altogether a very different kind of procedure from that required in an ordinary case of stricture treated by dilatation.

In Case 6, in which a very bad stricture in the bulbous urethra was complicated by multiple strictures in the penile portion, I slit up the urethra from the membranous portion to within an inch of the meatus, dividing, of course, the scrotum

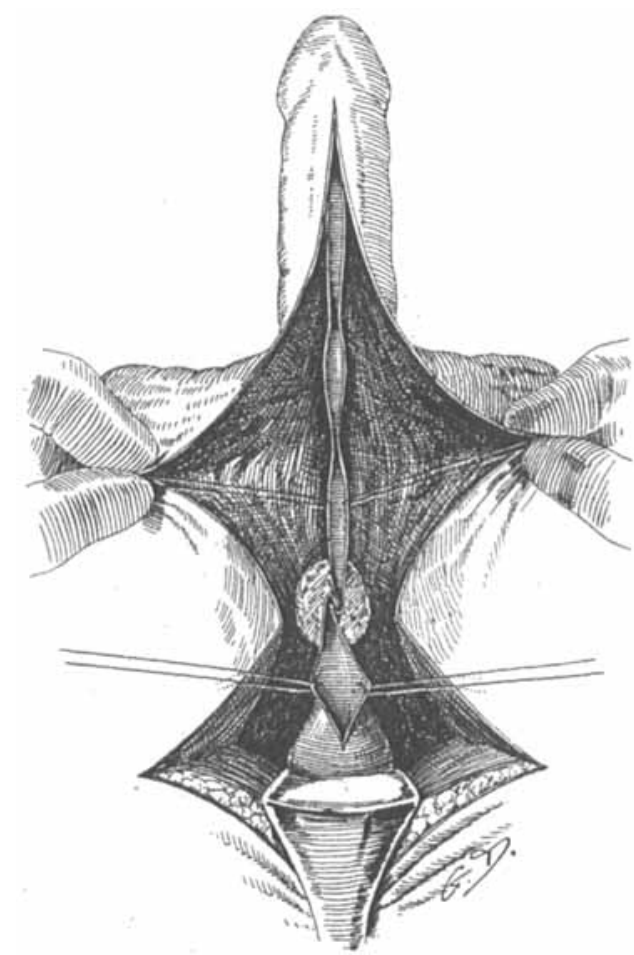

Frg. 211.-Multiple strictures in the penile urethre, exposed by dividing the scrotum and penis; a co-existing stricture of the bulbous urethra is exposed as before. (Fig. 211). The stricture of the bulb was excised, and the penile strictures

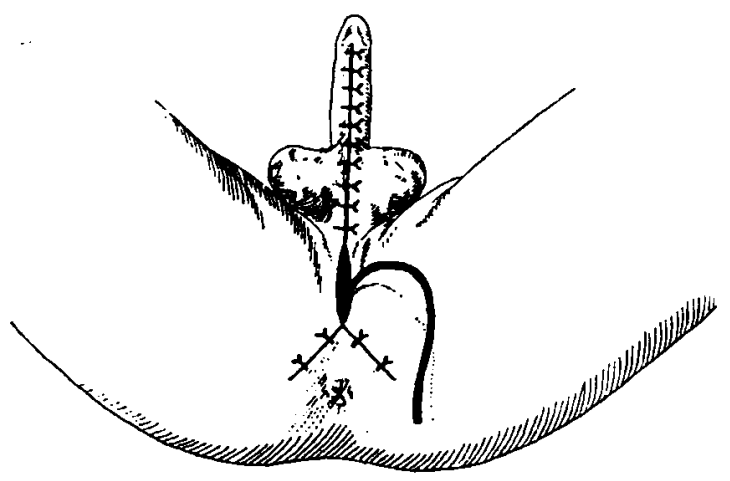

Fio. 212.-Suture of the wounds shown in the previous figure; in the penile portion the margins of the urethra are included in the skin suture. treated plastically: No special sutures were put into the urethra other than those at the seat of excision; only in the penile portion the lateral margins of the urethra were caught up and approximated by inclusion in the skin sutures. The skin and scrotum were sutured, and healing took place uneventfully throughout (Fig. 212). A full-sized instrument was easily passed for the first time five weeks after the operation. The case was also complicated

by a right inguinal hernia, which was operated upon at the same time. 


\section{THE BRITISH JOURNAL OF SURGERY}

In conclusion, I would endeavour to indicate what seems to be the exact place of this operation in the surgery of urethral stricture; and in order not to appear to make exaggerated claims for it, I will simply indicate its position in my own practice.

1. In all cases of stricture that are not easily managed by dilatation, excision of the stricture is advised.

2. In all cases of stricture that are easily managed by dilatation, no further operation is advised; nevertheless, even for patients in this category who may desire to be rid of even a moderate stricture, the operation, in my opinion, enables us to offer them a safe and efficient remedy.

3. It will thus be seen that this operation should, in my opinion, entirely supersede both internal and external urethrotomy as now performed.

\section{REPORT OF EIGHT CASES.}

(Exclusive of the case of Rupture of the Crethra described above.)

Case 1.-E. S., age 18. Admitted to Alfred Hospital, September, 1911. Had suffered from trouble with micturition since he was eight years old; possible cause, infection in childhood. Twice during the last two years has been under treatment at the Alfred Hospital for complete retention, which on both occasions had been very difficult to relieve. The stricture was excised on September 14, and the hypospadias operation performed. This was closed in 1012, as there was no sign of return of stricture, and after this had been done no instrument was introduced for twelve months, when a full-sized bougie passed easily.

Case 2.-A. C., age 39. Admitted to Alfred Hospital, December, 1912. Duration of stricture, eighteen years. On admission, passed water with great difficulty and straining. Stricture excised ; the first.instrument (a full-sized one) was passed quite easily seven weeks after the operation. He comes every three months for inspection. He has married and become a father since the operation.

Case 3.-E. B., age 48. Admitted to Alfred Hospital, December, 1912. Recent extravasation, for which perineum had been incised before admission to hospital; passing all water through perineal wound; there was present also a phlegmonous swelling (urinary infiltration) in hypogastrium to right of mid-line. Stricture excised, December 31. First instrument passed April 18, 1913, three and a half months after operation, when full-sized bougie passed with ease.

Case 4.-A. D., age 48. Admitted to Alfred Hospital, June, 1913. Gonorrhoea twenty-seven years ago ; stricture evident for last four years. On admission, urine passed guttatim; no instrument could be passed. Excision of stricture, June, 1913. First bougie passed a month after operation, when Lister 9-12 passed easily; next instrument three months later, when Lister 11-14 passed easily.

Case 5.-S. B., age 55. Admitted to Alfred Hospital, October, 1918. Had internal urethrotomy performed twelve years previously. For the last six years had been treated every few weeks at the Alfred Hospital by dilatation; stricture refractory and difficult to treat. Excision performed, October 28. First instrument passed at end of January, 1914, when full-sized bougie passed easily.

Case 6.-The case described above, with multiple strictures and right inguinal hernia, in which almost the entire length of the urethra was slit up, illustrated in Figs. 211 and 212.

The two following reports have been kindly supplied by my colleague, Mr. Fay Maclure, assistant surgeon to the Alfred Hospital.

Case 7. $\rightarrow$ S. R., age 50. Gonorrhcea thirty years ago. Difficulty in micturition ten years ; urine now escapes in a dribble only. There is a hard cylindrical mass in the perineum, about two inches in length. Attempts with filiform and other bougies were unsuccessful. 


\section{TREATMENT OF URETHRAL STRICTURE 383}

Operation of excision according to method described above. Catheter removed on the fifth day. Wound closed seventeenth day after operation. One month after leaving hospital, Lister bougie 10-13 passed readily; five months later the same instrument was again passed with equal facility.

Case 8.-B. S., age 60. Difficulty in micturition forty years; great difficulty for twenty years ; dribbling (no stream) for five years. Efforts to pass the stricture with bougies failed.

Operation, January 9, 191..-Excision as described above. First instrument passed five months later, when Iister 12-15 passed easily.

Note on Perineal Drainage of the Bladder.-Perineal drainage, as ordinarily performed by the introduction of a catheter into the bladder through a median perineal incision, is notoriously unsatisfactory for the purpose of protecting plastic operations on the urethra from urinary contamination; most surgeons, in fact, prefer suprapubic drainage for the attainment of such an object. Where, however, the urethra has been exposed and widely opened up in the way I have described, with detachment of the external sphincter from the bulbocavernosus muscle, bladder drainage by means of a catheter introduced through the membranoprostatic urethra is completely efficient; but I have thought it necessary to lay stress on the non-suturing of the perineal wound immediately in front of the catheter; this is an obvious precaution against the escape of any urine past the catheter into the urethra in front of it. 